\title{
Thin-walled parts flexible fixture system rigid positioning support research
}

\author{
Chen Yong ${ }^{1, a}$, Jiang Yongmin ${ }^{2, b}$ \\ 1,2 Department of Mechanical Engineering, Chengdu industrial University, Chengdu 610031 China \\ a 923985767@qq.com, b 825182196@qq.com,
}

Keywords: The flexible fixture, clamping rigidity, surface positioning, supporting distribution.

Abstract. For aircraft large thin-walled flexible fixture locating surface is small, insufficient clamping force, clamping rigidity is not enough, not suitable for milling, in order to solve the problem of cutting force and installation stiffness do not match, approximate surface developed the intelligent flexible process equipment support system. Using approximate surface orientation, local clamping. Through the analysis of system structure and operation principle, has established the mathematical model of the proposed adaptive optimization method such as curvature surface positioning. The method according to the given workpiece curvature, selection of curvature approximation of the bearing surface, adaptively adjust positioning/support distribution of flexible fixture system, guarantee the deformation reaching a minimum overall. Example shows that this method can make flexible fixture system supporting distribution in the optimal state, the clamping stiffness meet the requirements of milling, achieve the best utilization of resources, meet the demand of vehicle high speed and high precision machining of large thin wall parts. :

\section{Introduction}

In theory, USES the space more adjustable support flexible fixture positioning, implements multiple parts a tooling, reduce the tooling cost. In fact, the flexible fixture is space point positioning, no bearing surface of joint, clamping force is not enough, for milling cutting force is bigger, do not apply. To aircraft skin flexible tooling practical application, it is necessary to study the flexible fixture positioning and clamping force, improve the practicality.

In recent years, many scholars of machining deformation control problem of large thin wall parts were studied, with the continuous improvement of automation level, more flexible fixture system is becoming a hot spot of research, it can effectively solve large thin wall parts without cutting force under the condition of the positioning of the problem. Under the condition of considering small cutting force, or under their own gravity, support the position and deformation or the relationship between the positioning error, the more flexible fixture system optimization support method to get the attention. Zhou Kai and Zhang etc. [1] through the finite element method to analyze deformation of thin-walled multi-point flexible manufacturing, and summarize the regularity of deformation; When Zhou Kai and lu, such as [2-4] proposed two kinds of adaptive optimization method and genetic algorithm method to optimize the clamping layout, optimization analysis to obtain the optimal layout, make the best use of resources, and satisfy the processing requirements. Has processed parts surrounding contour and parts of the hole is a large deformation, the aircraft aerodynamic and stealth performance are caused great impact.

This paper introduces a flexible positioning a tooling equipment and special tooling, a flexible and certain support rigidity, can adapt to the milling processing, there are two main technical way to solve this problem: (1) the rigid way, using similar curvature, and rigid good cylinder as the local bearing surface of workpiece machining parts. (2) the flexible way, through the adjustment and control means of dynamically generated positioning support needed for the bearing surface of cylinder, cylinder bearing surface can be replaced. So a tooling can be used for different parts of processing, greatly improve the flexible and rigid. . 


\section{The flexible system of vacuum adsorption clamping}

The composition of flexible fixture system . Aimed at the specialties of the spacecraft large thin-walled curved surface parts processing, the development of the intelligent flexible fixture based on robot operating system, its basic structure is shown in figure 1. Its characteristics: the more moving beam units mounted on the pedestal part 24 , each moving beam components are along the $\mathrm{x}$ direction; Moving on the beam is equipped with multiple sliding parts 5, each sliding parts are along the y direction. Telescopic unit mounted on the sliding part 6 , the universal vacuum at the top of the telescopic unit will lead to the first eight, along the $\mathrm{Z}$ direction.

The system could be used under computer control, according to the need to generate.

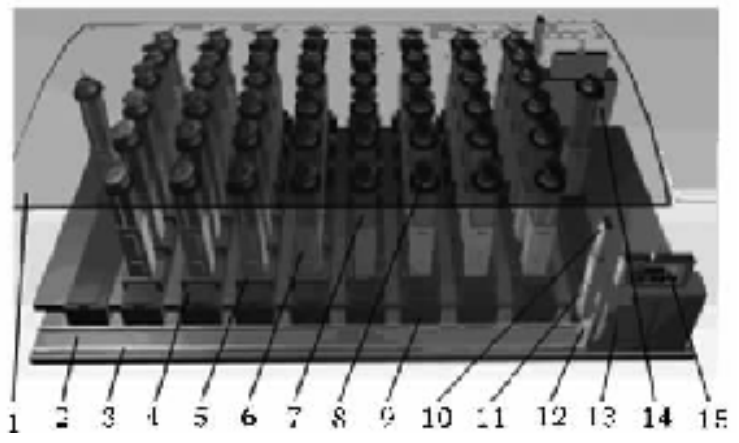

1 - artifacts;2 base;3 - guide rail;4 - moving beam;5 - sliding parts; 6 - telescopic unit; 7 supporting rod;8 - vacuum head;9 a locking block;Forearm 1110 robot manipulator;12 small manipulator;13.14 a robot;15 - controller.

Figure 1, flexible fixture system structure

I In order to reduce the deformation, it is necessary to improve the density of bearing for supporting unit small volume as much as possible. Due to the servo motor and drive to take up larger space position, so can't use conventional technologies such as leadscrew nut controlled worktable implementation of moving beam and sliding driver.

To solve this problem, it is used the combination of centralized drive and distribution of drive. Both sides of the base part 2 in figure 1 system installed two robot 13 and 14, can be synchronous movement along the $\mathrm{x}$ direction. Mobile robot can move the unit in the $\mathrm{x}$, $\mathrm{y}$ direction. The medial aspect of the each robot is equipped with two small manipulator, respectively used to beam along the $\mathrm{x}$ direction and lock. Robot front end is equipped with large manipulator 11, but through the rotation, scaling, scraping, along y direction sliding parts 5, and hydraulic lock, telescopic unit to drive after vacuum suction lift along the $\mathrm{Z}$ direction, the resulting specified positioning/support array.

\section{The objective function of optimization method}

The objective function for optimization method. Positioning/support distribution of flexible fixture system need to adaptively according to the machining deformation of workpiece in the process of adjustment, adjust the goal is the overall deformation is minimal. Optimization process is to change the design variables according to the target state, which requires the introduction of design sensitivity. Design sensitivity analysis is to calculate the objective function and constraint function relative to the design variables of derivative. Design sensitivity can provide designers the message: in a given constraint conditions, how to change the design variables to effectively improve the objective function. .

$$
\frac{\partial \xi}{\partial V}=\frac{g\left(V^{+}\right)-g\left(V^{i}\right)}{2 \Delta y_{i}}
$$

Mechanics analysis of flexible fixture . Analysis of flexible tooling support, support is limited, most of the places in a suspended state, the main factors influencing the location accuracy is: part weight distribution of force and law to the cutting force produced by degrees. Machining parts in the process of elastic deformation is the main factors affecting the quality of parts processing, parts somewhere elastic deformation caused by the cutting force components mainly for cutting force along the normal direction at that point in the component. In processing on the trajectory caused by gravity force distribution around the biggest degree as the objective function, or by the cutting force along the point method to the maximum number of elastic deformation caused by the weight of parts 
as objective function and constraint conditions including the system structure and process conditions constraints. Weight of simplified as below:

The maximum deflection of beam

$E I w_{\max }=\frac{1}{24} q\left[\frac{l}{2}\right]^{4}-\frac{q l}{12}\left[\frac{l}{2}\right]^{3}+\frac{q^{2}}{24}\left[\frac{l}{2}\right]=\frac{5 b h l^{2}}{384 E I}$

$E$ for the elastic modulus of aluminum, for engineering structure using aluminum, $E=720000$ $\mathrm{N} /$ was. I used for cross section moment, $\mathrm{I}=\mathrm{bh} 2 / 12, \mathrm{q}=\mathrm{bdh} \mathrm{b}$ width, thickness of $\mathrm{h}, \mathrm{d}$, the calculating formula of $\mathrm{w}:=$ bh squared square and rectangular $\mathrm{w} / 6$

Across the maximum deflection under a concentrated load in the span of beam, the calculation formula:

$\mathrm{Z}_{\max }=8 p l^{3} /(384 E I)=p l^{3} /(48 E I)$.

Type: Zmax for maximum deflection of beam span $(\mathrm{mm}) . \quad P$ is the sum of the concentrated load standard values $(\mathrm{kn})$ for aluminum elastic modulus $\mathrm{E}, \mathrm{E}=1000000 \mathrm{~N} /$ was. I used for steel section moment, can look up table (mm4).

\section{The flexible system of workpiece deformation}

The example analysis . In order to verify the application effect of this method, in view of the number of aircraft, the optimization of large thin wall parts. Figure 2 for parts and around the degree of spatial distribution, shape size is $1800 \mathrm{~mm}$ x $1100 \mathrm{~mm}$, thickness is $5 \mathrm{~mm}$, material for aluminum alloy
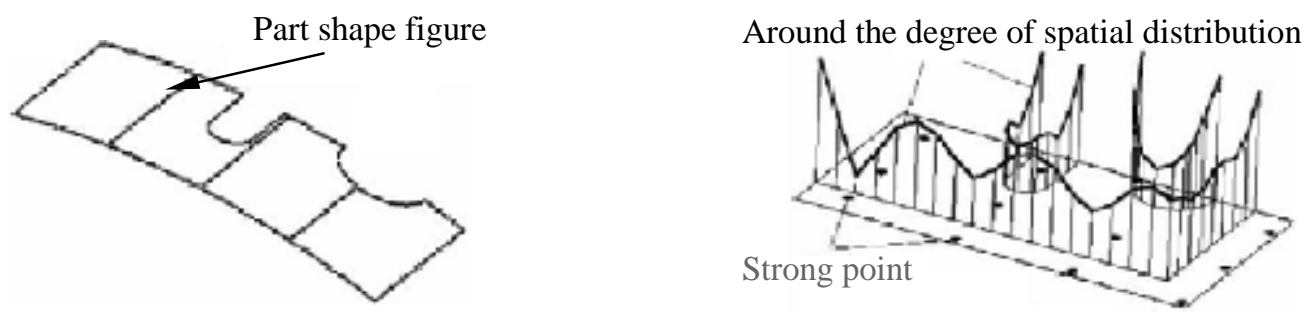

Figure 2 parts and around the degree of spatial distribution

As can be seen from the figure 2: in the processing part of the cutting, as there is force, deformation is bigger; Away from the point of part deformation; Conclusion is the need to increase the anchoring, resist cutting force, reduce the deformation.

The specific parameters of flexible fixture system: supporting the sphere: $=19 \mathrm{~mm}$, moving beam number $\mathrm{m}=8$, every move on the beam positioning/support unit number $\mathrm{n}=6$. Supporting point on the adjacent two moving beam in the $\mathrm{x}$ direction from the minimum $\mathrm{d}_{\mathrm{xmin}}=150 \mathrm{~mm}$, with the move beam on the adjacent two supporting points in $\mathrm{y}$, direction distance minimum $\mathrm{d}_{\mathrm{ymin}}=150 \mathrm{~mm}$.

Request processing deformation is of less than $0.34 \mathrm{~mm}$ in locus on the maximum. Application of adaptive optimization method, adjust bearing distribution after 25 times, get the results meet the requirements as shown in figure 3. Correspond with below the position of the workpiece, and the solid line shows the processing trajectory, each point on the dotted line processing locus, the deformation of the black dots represent bearing point. Under this kind of bearing distribution, processing and track the maximum deformation of $0.328 \mathrm{~mm}$.

\section{The exploration of flexible approximate rigid fixed bearing surface}

Approximate bearing surface problems . Increase point in front of the flexible system is limited and difficult. The traditional specialized equipment has no more flexible and convenient features. In order to make tooling have both flexible and rigid, explore the approximate curved rigid support.

The thickness of the bearing surface is assumed to be $25 \mathrm{~mm}$ cylinder, has certain rigidity. Outside the circle radius is $425 \mathrm{~mm}$, the radius of $475 \mathrm{~mm}$, thickness is $2 \mathrm{~mm}$ skin parts joint outer circular surface, observation of joint, as shown in figure 4, within the scope of the $400 \mathrm{~mm}$, maximum joint 
size is $5.84 \mathrm{~mm}$, not Angle difference: $5.84 / 200=0.029$, the deformation of $3 \%$. Belong to the small deformation, elastic deformation range. If at the time of processing, exerting force, will be tight, my wallet skin elastic deformation, completely joint bearing surface is $425 \mathrm{~mm}$ in radius, after processing, skin parts will return to its original shape. Such recoverable in part deformation state, created a completely rigid support conditions, namely satisfy certain flexibility and satisfy certain rigidity.

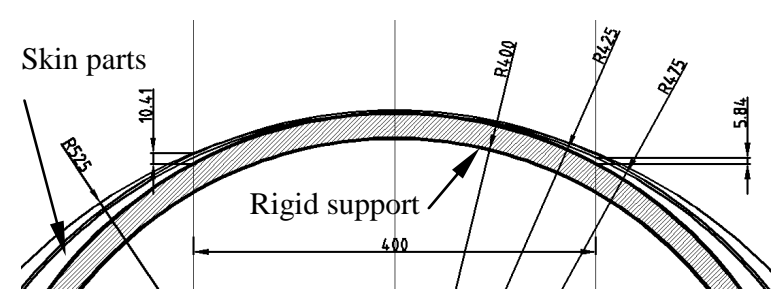

Figure 3 fit curvature radius is $100 \mathrm{~mm}$

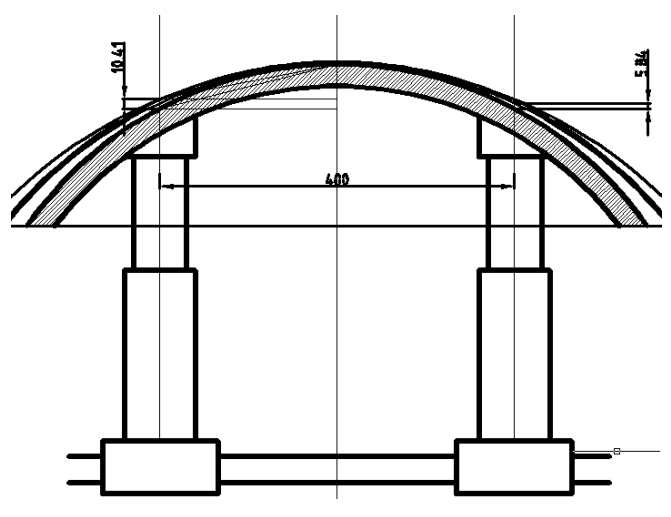

Figure 4, supporting flexible support below

When the radius of curvature difference is $100 \mathrm{~mm}$, as shown in figure 4 , the bearing surface which radius is $525 \mathrm{~mm}$ joints at cylinder which radius is $425 \mathrm{~mm}$, no fit size is $10.41 \mathrm{~mm}$, Angle difference: $10.41 / 200=0.052$, the deformation of $5 \%$. Also belong to small deformation in the elastic deformation range.

Even radius is $100 \mathrm{~mm}, 400 \mathrm{~mm}$ maximum Angle error is 5\%, within the scope of tiny deformation Angle. Show the approximate curvature on the surface of the support the range is very large.

\section{Conclusion}

Main problem of flexible fixture system is limited support density, poor rigidity, not suitable for milling. flexible assembly tooling can reduce the number of traditional rigid tooling in great quantities, shorten the tooling time to prepare, thus greatly shorten the development cycle of the aircraft, improved the production efficiency.

1) Manufacturing period shorten developed a set of aircraft skin flexible fixtures, solving a set of tools can only process one skin components.

2) Using approximation curvature support plate, can satisfy a certain flexibility and satisfies the requirement of certain rigidity.

3) Within the scope of the elastic deformation, an approximation curvature radius of support plate can be suitable for a set of artifacts, radius range is larger.

\section{Acknowledgment}

The authors greatly appreciate the following sponsors for their support to the study:

The natural science foundation of education committee of Sichuan province, (08zb070).

\section{References}

[1] Zhou kai and Lu. Tooling robot research of wireless control system [J]. Manufacturing technology and machine tools, 2009. (3) : p. 14-17.

[2] Zhou kai and Wu. Since the mountain song and thin walled workpiece machining flexible positioning method research [J]. Manufacturing technology and machine tools, 2008 . (10) : 113-117. 